\title{
COHOMOLOGY GROUPS OF COMMUTATIVE BANACH ALGEBRAS $\left({ }^{1}\right)$
}

\author{
BY \\ HERBERT KAMOWITZ
}

Introduction. The aim of this paper is to extend the cohomology theory of Hochschild to commutative Banach algebras and to investigate some consequences that may be derived from this extension. Suppose $A$ is an associative algebra and $P$ is an $A$-module. Then in [5]( $\left.{ }^{2}\right)$ Hochschild attached to this pair, $A, P$ a sequence of abelian groups $H^{k}(A, P), k=1,2, \cdots$. These groups are called cohomology groups.

Three principal results of [5] are the following.

THEOREM A. In order that the finite dimensional associative algebra $A$ be separable, i.e. semi-simple over every algebraic extension of the ground field, it is necessary and sufficient that $H^{k}(A, P)$ vanish for all two-sided $A$-modules. $P$ and all positive integers $k$.

THEOREM B. If $A$ is a finite dimensional associative algebra, then a necessary and sufficient condition that $H^{2}(A, P)$ should vanish for all $A$-modules $P$ is that for every finite dimensional algebra $B$ with radical $R$, if $B / R=A$, then there exists a subalgebra $A^{\prime}$ of $B$ with $B=A^{\prime} \oplus R$.

Theorems A and B are then used to give another proof of the Wedderburn principal theorem.

TheOREM C (WEDDERBURN). Let $B$ be a finite dimensional associative algebra and $R$ its radical. If $A=B / R$ is separable, then there exists a subalgebra $A^{\prime}$ of $B$ with $B=A^{\prime} \oplus R$.

Without topological considerations if we do not require the algebra $A$ to be finite dimensional, then Theorems $\mathrm{A}$ and $\mathrm{C}$ are no longer valid. For a discussion of this we refer the reader to [9] and [11].

When considering the Wedderburn principal theorem for Banach algebras we shall require that the subalgebra $A^{\prime}$ of $B$ be closed and hence that $A^{\prime}$ is both isomorphic and norm-equivalent to $A$. In [4], Feldman has shown that there exists a Banach algebra $B$ with one-dimensional radical $R$ such that $B / R=l^{2}$ (the algebra of square summable sequences with coordinate-wise

Presented to the Society, April 16, 1960; received by the editors October 18, 1960 and, in revised form, April 16, 1961.

(1) This paper represents a portion of a thesis submitted to Brown University in partial fulfillment of the requirements for the degree of Doctor of Philosophy. The author wishes to thank Professor John Wermer for his generous help in preparation of this paper.

(2) Numbers in brackets refer to references cited at the end of the paper. 
multiplication) but with no closed subalgebra $A^{\prime}$ of $B$ satisfying $B=A^{\prime} \oplus R$.

Bade and Curtis [2] have considered the Wedderburn principal theorem in the case $A=C(X)$, the Banach algebra of complex-valued continuous functions on a compact Hausdorff space $X$. Among other things they show the following. (1) If $X$ is extremely disconnected and $B / R=C(X)$ then there exists a unique closed subalgebra $A^{\prime}$ of $B$ with $B=A^{\prime} \oplus R$. (2) If the radical $R$ of $B$ is finite dimensional and $B / R=C(X)$, any compact $X$, then there exists a unique closed subalgebra $A^{\prime}$ of $B$ with $B=A^{\prime} \oplus R$. (3) If $B / R=C(X)$ and if there exists a closed subalgebra $A^{\prime}$ such that $B=A^{\prime} \oplus R$, then $A^{\prime}$ is unique. (4) There exists a Banach algebra $B$ with radical $R$ and $B / R=(c)$, the algebra of convergent sequences, such that no closed subalgebra $A^{\prime}$ satisfies $B=A^{\prime} \oplus R$.

In this paper we consider commutative Banach algebras and define onesided "normed" $A$-modules. We shall see that Theorem A does not extend, but that the connection between the vanishing of $H^{2}(A, M)$ for all normed $A$-modules $M$ and the Wedderburn principal theorem carries over to the present case almost in its entirety. $\$ 3$ is devoted to a generalization of Theorem B.

In $\$ 4$ it is shown that if $X$ is a locally compact Hausdorff space, then for all normed $C(X)$-modules $M, H^{1}(C(X), M)=\{0\}$ and $H^{2}(C(X), M)=\{0\}$. As a result the following Wedderburn type theorem holds. Suppose $B$ is a commutative Banach algebra, $X$ is a locally compact Hausdorff space, $B / R=C(X), R$ is nilpotent and $R$ and the closure of every even power of $R$ have a Banach space complement in $B$. Then there exists a unique closed subalgebra $C$ of $B$ with $B=C \oplus R$.

\section{Basic definitions.}

Definition 1.1. Suppose $A$ is a commutative Banach algebra. Then $(M, \cdot)$ is a normed $A$-module if, and only if,

(i) $M$ is a Banach space,

(ii) $(M, \cdot)$ is a left $A$-module in the usual sense,

(iii) $\sup \{\|a \cdot m\| \mid\|a\|=\|m\|=1\}<\infty$.

In case $(M, \cdot)$ is a normed $A$-module, $\|M\|$ will denote $\sup \{\|a \cdot m\|\|\| a \|$ $=\|m\|=1\}$.

Since we shall be dealing principally with one-sided left modules the adjectives "one-sided" and "left" will usually be omitted. Two extreme but useful types of modules are described in the following definition.

Definition 1.2. Suppose $A$ is a commutative Banach algebra and $(M, \cdot)$ is a normed $A$-module. Then

(i) $(M, \cdot)$ is unital if, and only if, $m \in M$ and $a \cdot m=0$ for all $a \in A$ imply $m=0$.

(ii) $(M, \cdot)$ is a zero $A$-module if, and only if, $a \cdot m=0$ for all $a \in A$ and $m \in M$.

It is easy to see that if $A$ has an identity then Definition 1.2(i) is equiv- 
alent to the usual definition of unital (unitary) module. If $A$ is a commutative Banach algebra and $(M, \cdot)$ a normed $A$-module, then one can use the fact that each $m \in M$ may be written as $m=1 \cdot m+(m-1 \cdot m)$ to prove the following.

TheOREM 1.1. Suppose $A$ is a commutative Banach algebra with 1 as the identity and $(M, \cdot)$ is a normed $A$-module. Let $M_{0}=\{m \in M \mid x \cdot m=0$ for all $x \in A\}$ and $M_{1}=\{1 \cdot m \mid m \in M\}$. Then $\left(M_{0}, \cdot\right)$ is a zero normed $A$-module, $\left(M_{1}, \cdot\right)$ is a unital normed $A$-module and $M=M_{0} \oplus M_{1}$.

If $A$ lacks an identity, then $A$-modules need not be decomposable as a direct sum of a zero normed $A$-module and unital normed $A$-module. Indeed let $A$ be the algebra of absolutely convergent sequences on the integers with addition and multiplication defined coordinate-wise. Let $M=L^{1}(0,2 \pi)$. If $a \in A$ and $f \in L^{1}(0,2 \pi)$ let $a \cdot f$ be that function in $L^{1}(0,2 \pi)$ whose Fourier transform is given by $(a \cdot f){ }_{n}=0$ if $n<0$ and $(a \cdot f)^{\wedge}{ }_{n}=a_{n} f_{n}$ for $n \geqq 0$. Then $M_{0}=\left\{f \in L^{1}(0,2 \pi) \mid f_{n}=0\right.$ for $\left.n \geqq 0\right\}$. But $M_{0}$ has no complement in $M$ [8].

DEFINITION 1.3. Let $I$ be a bounded isomorphism of the commutative Banach algebra $A$ onto the commutative Banach algebra $A^{\prime}$. The normed $A$-module $(M, \cdot)$ and normed $A^{\prime}$-module $\left(M^{\prime}, \cdot^{\prime}\right)$ are said to be isomorphic provided there is a bounded isomorphism $I^{\prime}$ of $M$ onto $M^{\prime}$ such that for all $a \in A$ and $m \in M, I^{\prime}(a \cdot m)=I(a) \cdot{ }^{\prime} I^{\prime}(m)$.

We now list a series of definitions relating to cohomology groups on which most of the results are based. In Definitions 1.4 through 1.8, $A$ will always represent a commutative Banach algebra, $(M, \cdot)$ a normed $A$-module and $k$ an element of $\omega$, the set of positive integers.

Definition 1.4. A $(k, M)$-cochain of $A$ is a $k$-linear map of $A \times \cdots \times A$ ( $k A$ 's) into $M$. The coboundary operator $\delta$ which maps $(k, M)$-cochains of $A$ into $(k+1, M)$-cochains of $A$ is defined for each $(k, M)$-cochain $f$ by

$$
\begin{aligned}
\delta f\left(a_{1}, \cdots, a_{k+1}\right)= & a_{1} \cdot f\left(a_{2}, \cdots, a_{k+1}\right)+\sum_{i=1}^{k}(-1)^{i} f\left(a_{1}, \cdots, a_{i} a_{i+1}, \cdots, a_{k+1}\right) \\
& +(-1)^{k+1} a_{k+1} \cdot f\left(a_{1}, \cdots, a_{k}\right) .
\end{aligned}
$$

Definition 1.5. If $f$ is a $(k, M)$-cochain of $A$ and $\delta f=0$, then $f$ is a $(k, M)$ cocycle of $A$. If $k>1$, then the cochain $f$ is a $(k, M)$-coboundary of $A$ provided $f=\delta g$ for some $(k-1, M)$-cochain $g$ of $A$. The $(1, M)$-cochain $f$ is a $(1, M)$ coboundary of $A$ provided $f=0$.

REMARK. It is shown in [5] that $\delta \delta f=0$ for all cochains $f$.

Definition 1.6. A $(k, M)$-cochain of $A$ is said to be bounded provided there exists $c>0$ such that $\left\|f\left(a_{1}, \cdots, a_{k}\right)\right\| \leqq c\left\|a_{1}\right\| \cdots\left\|a_{k}\right\|$ for all $a_{1}, \cdots, a_{k}$ $\in A$. If $f$ is a bounded $(k, M)$-cochain of $A$ then

$$
\begin{aligned}
\|f\| & =\inf \left\{c \mid\left\|f\left(a_{1}, \cdots, a_{k}\right)\right\| \leqq c\left\|a_{1}\right\| \cdots\left\|a_{k}\right\|, a_{i} \in A\right\} \\
& =\sup \left\{\left\|f\left(a_{1}, \cdots, a_{k}\right)\right\| \mid a_{i} \subseteq A, i=1, \cdots, k \text { and }\left\|a_{i}\right\|=1\right\} .
\end{aligned}
$$


Definition 1.7. We let $C^{k}(A, M)$ be the set of bounded $(k, M)$-cochains of $A . Z^{k}(A, M)$ is the set of bounded $(k, M)$-cocycles of $A$ and $B^{k}(A, M)$ is the set of bounded $(k, M)$-coboundaries of $A$.

Under addition each of the three sets just defined is a commutative group.

Definition 1.8. The factor group $Z^{k}(A, M) / B^{k}(A, M)$ is the $k$-dimensional cohomology group of $A$ into $M$ and is denoted by $H^{k}(A, M)$. Elements of $H^{k}(A, M)$ are called $(k, M)$-cohomology classes of $A$.

Usually $(1, M)$-cocycles are called derivations and we shall use the two terms interchangeably. Then $H^{1}(A, M)$ is the set of bounded derivations of $A$ into $M$ when we make the convention that $B^{1}(A, M)=\{0\}$. This paper will deal almost exclusively with one- and two-dimensional cohomology groups.

Since $\delta$ is a bounded linear map of $C^{k}(A, M)$ into $C^{k+1}(A, M)$, we have the following.

TheOREM 1.2. Suppose $A$ is a commutative Banach algebra and $(M, \cdot)$ is a normed $A$-module. Then for $k \in \omega, C^{k}(A, M)$ is a Banach space and $Z^{k}(A, M)$ is a closed subspace of $C^{k}(A, M)$. The norm is that given in Definition 1.6.

REMARK. Example 2.1 will show that $B^{2}(A, M)$ is not necessarily closed in $C^{2}(A, M)$ and thus cohomology groups need not be Banach spaces.

We state without proof two theorems which may be easily verified.

Theorem 1.3. Suppose $A$ is a commutative Banach algebra, $(M, \cdot),\left(M_{1}, \cdot\right)$ and $\left(M_{2}, \cdot\right)$ are normed $A$-modules and $M=M_{1} \oplus M_{2}$. Then for all $k \in \omega$, $H^{k}(A, M)$ is isomorphic to $H^{k}\left(A, M_{1}\right) \oplus H^{k}\left(A, M_{2}\right)$.

TheOREM 1.4. Suppose $A$ and $A^{\prime}$ are isomorphic commutative Banach algebras and the normed $A$-module $(M, \cdot)$ is isomorphic to the normed $A^{\prime}$-module $\left(M^{\prime}, \cdot^{\prime}\right)$. Then for each $k \in \omega$, each of the following is a pair of isomorphic groups: $\left(C^{k}(A, M), C^{k}\left(A^{\prime}, M^{\prime}\right)\right),\left(Z^{k}(A, M), Z^{k}\left(A^{\prime}, M^{\prime}\right)\right),\left(B^{k}(A, M), B^{k}\left(A^{\prime}, M^{\prime}\right)\right)$ and $\left(H^{k}(A, M), H^{k}\left(A^{\prime}, M^{\prime}\right)\right)$.

THEOREM 1.5. If $A$ is a commutative Banach algebra with identity 1 and $(M, \cdot)$ is a zero normed $A$-module, then $H^{k}(A, M)=\{0\}$ for all $k \in \omega$.

Proof. Suppose $f \in Z^{k}(A, M)$. A straightforward computation shows that the $(k-1)$-linear map $g$ defined by $g\left(x_{1}, \cdots, x_{k-1}\right)=-f\left(1, x_{1}, \cdots, x_{k-1}\right)$ is bounded and satisfies $f=\delta g$.

As a result of Theorems $1.1,1.3$ and 1.5 the vanishing of cohomology groups of algebras with an identity depends only on whether the corresponding groups vanish over unital modules.

Now let $A$ be a commutative Banach algebra without an identity. Then we let $A_{0}$ be the algebra obtained by adjoining a unit to $A$ in the usual way [7, p. 59]. Elements of $A_{0}$ are denoted by $x_{\lambda}$ or $\langle x, \lambda\rangle$ for $x \in A$ and $\lambda$ a scalar. The multiplicative identity $\langle 0,1\rangle$ is denoted by $e$. The norm of $x_{\lambda}$ is $\|x\|+|\lambda|$. 
Definition 1.9. Suppose $A$ is a commutative Banach algebra with no identity and $(M, \cdot)$ is a normed $A$-module. Then we say that the normed $A_{e}$-module $(M, \cdot)$ is an extension in the canonical manner provided $x_{\lambda} \cdot m$ $=x \cdot m+\lambda m$ for all $m \in M$ and $x_{\lambda} \in A_{\text {e }}$.

The extension in the canonical manner is unital.

THeOREM 1.6. Suppose $A$ is a commutative Banach algebra without an identity and $(M, \cdot)$ is a normed $A$-module. Then when $(M, \cdot)$ is considered as an $A_{\mathrm{e}}$-module in the canonical manner, the following hold:

(i) $H^{1}\left(A_{\odot}, M\right)=\{0\}$ if, and only if, $H^{1}(A, M)=\{0\}$.

(ii) $H^{2}\left(A_{\bullet}, M\right)=\{0\}$ if, and only if, $H^{2}(A, M)=\{0\}$.

Proof. If $d$ is a bounded derivation of $A$ into the normed $A$-module $(M, \cdot)$, then the map $d^{\prime}: d^{\prime}\left(x_{\lambda}\right)=d(x)$ for $x_{\lambda} \in A_{e}$ is a bounded derivation of $A_{\bullet}$ into the $A_{\mathrm{e}}$-module $(M, \cdot)$. Conversely if $d^{\prime}$ is a bounded derivation of $A_{\bullet}$ into $M$, then $d: d(x)=d^{\prime}\left(x_{0}\right)$ is a bounded derivation of $A$ into $M$. Clearly in each case $d=0$ if, and only if, $d^{\prime}=0$ and so $H^{1}(A, M)=\{0\}$ if, and only if, $H^{1}\left(A_{\theta}, M\right)$ $=\{0\}$.

If $f$ is a bounded $(2, M)$-cocycle of $A$, then $f^{\prime}: f^{\prime}\left(x_{\lambda}, y_{\mu}\right)=f(x, y)$ is a bounded $(2, M)$-cocycle of $A_{e}$ which is a bounded $(2, M)$-coboundary of $A_{\bullet}$ if $f$ is a bounded $(2, M)$-coboundary of $A$. Indeed if $f=\delta g$, then $f^{\prime}=\delta g^{\prime}$, where $g^{\prime}\left(x_{\lambda}\right)=g(x)$. Conversely if $f^{\prime}$ is a bounded $(2, M)$-cocycle of $A_{\boldsymbol{e}}$, then $f: f(x, y)$ $=f^{\prime}\left(x_{0}, y_{0}\right)$ is a bounded $(2, M)$-cocycle of $A$ and is a $(2, M)$-coboundary of $A$ if $f^{\prime}$ is a $(2, M)$-coboundary of $A_{\text {e. }}$. For if $f^{\prime}=\delta g^{\prime}$, let $g(x)=g^{\prime}\left(x_{0}\right)$. Then $f=\delta g$. Thus $H^{2}(A, M)=\{0\}$ if, and only if, $H^{2}\left(A_{\bullet}, M\right)=\{0\}$.

2. Some examples. As we noted in the introduction, a basic theorem in [5] states that a necessary and sufficient condition that a finite dimensional algebra $A$ be separable is that $H^{k}(A, M)$ should vanish for all 2 -sided $A$ modules $(M, \cdot)$ and all $k \in \omega$.

We show now that if we add the condition that $A$ be a commutative Banach algebra, remove the requirement that $A$ be finite dimensional and consider normed $A$-modules and bounded cochains, then this result need no longer be true. We give three examples of Banach algebras $A$ and normed $A$-modules $(M, \cdot)$ for which $H^{2}(A, M) \neq\{0\}$.

EXAMPLE 2.1. Let $A=l^{2}$ and $\left\{e_{k}\right\}$ be a complete orthonormal basis. If $x=\sum_{k=1}^{\infty} x_{k} e_{k}$ and $y=\sum_{k=1}^{\infty} y_{k} e_{k}$, define multiplication in $A$ by $x y=\sum_{k=1}^{\infty} x_{k} y_{k} e_{k}$. Then $A$ is a commutative semi-simple Banach algebra. Let $K$ be a 1-dimensional Banach space and assume $\cdot$ is defined to make $(K, \cdot)$ a zero normed $A$-module.

We define the bounded $(2, K)$-cochains $f_{n}$ by $f_{n}(x, y)=\sum_{k=1}^{n} x_{k} y_{k} / k^{1 / 2}$. Then one sees that $f_{n} \in Z^{2}\left(l^{2}, K\right)$ for all $n \in \omega$. If $g(x)=-\sum_{k=1}^{n} x_{k} / k^{1 / 2}$, then $g_{n}$ is a bounded linear functional with $\delta g_{n}=f_{n}$ and so $f_{n} \in B^{2}\left(l^{2}, K\right)$. The $(2, K)$-cocycle $f: f(x, y)=\sum_{k=1}^{\infty} x_{k} y_{k} / k^{1 / 2}$ has the property that $\left\|f-f_{n}\right\| \rightarrow 0$ since $\left|f(x, y)-f_{n}(x, y)\right|=\left|\sum_{k=n+1}^{\infty} x_{k} y_{k} / k^{1 / 2}\right| \leqq(n+1)^{-1 / 2}\|x\|\|y\|$. However 
$f \notin B^{2}\left(l^{2}, K\right)$. Indeed should there exist a bounded linear functional $g$ on $l^{2}$ with $f=\delta g$, then we would have $g\left(e_{k}\right)=1 / k^{1 / 2}$. But $\left\{\sum_{k=1}^{n} x_{k} / k^{1 / 2}\right\}$ need not converge. Hence we have constructed a normed $l^{2}$-module $(K, \cdot)$ for which $H^{2}\left(l^{2}, K\right) \neq\{0\}$.

REMARK. Example 2.1 also shows that $B^{2}\left(l^{2}, K\right)$ is not closed.

REMARK. It is clear that a necessary condition that a $(2, M)$-cochain $f$ of a commutative Banach algebra $A$ be a $(2, M)$-coboundary is that $f(a, b)$ $=f(b, a)$ for all $a, b \in A$.

EXAMPLE 2.2. Let $A=C^{1}([-1,1] \times[-1,1])$, the algebra of continuous functions on the unit square having continuous first partial derivatives. The norm in $A$ is given by

$$
\|u\|_{A}=\|u\|_{\infty}+\left\|\frac{\partial u}{\partial x}\right\|_{\infty}+\left\|\frac{\partial u}{\partial y}\right\|_{\infty} .
$$

Let $(K, \cdot)$ be the 1-dimensional unital normed $A$-module with $u \cdot m=u(0,0) m$ for all $u \in A, m \in K$. A direct verification shows that the bounded $(2, K)$ cochain $f$ defined by

$$
f(u, v)=\left(\frac{\partial u}{\partial x}\right)_{(0,0)}\left(\frac{\partial v}{\partial y}\right)_{(0,0)}
$$

is a bounded $(2, K)$-cocycle of $A$. Since there exist $u, v \in A$ with $f(u, v) \neq f(v, u)$ we may conclude that $B^{2}(A, K) \neq C^{2}(A, K)$ and that $H^{2}(A, K) \neq\{0\}$.

In the following example we anticipate a result of $\$ 4$.

ExAmple 2.3. Let $A$ be the algebra of continuous functions on the unit circumference which are boundary values of functions analytic on the unit disc. For $u, v \in A$, define $f(u, v)$ by

$$
f(u, v)(t)=\left[\frac{u(t)-u(0)}{t}\right] v^{\prime}(0)+\left[\frac{v(t)-v(0)}{t}\right] u^{\prime}(0), \quad|t|=1 .
$$

Then $f(u, v) \in A$. A straightforward computation shows, too, that $f$ is a bounded $(2, A)$-cocycle of $A$.

We shall see (Lemma 4.5) that if $f=\delta g$, then for $2 \leqq n \in \omega$

$$
n u^{n-1} \cdot g(u)-g\left(u^{n}\right)=\sum_{i=0}^{n-2} u^{i} \cdot f\left(u, u^{n-i-1}\right) .
$$

Now $f(z, z)=2$ and $f\left(z, z^{k}\right)=z^{k-1}$ if $k>1$, where $z$ is the identity function. Then if $u=z$,

$$
n z^{n-1} \cdot g(z)--g\left(z^{n}\right)=(n-2) z^{n-2}+2 z^{n-2}=n z^{n-2} .
$$

Thus $g(z)(t)=(1 / n) t^{(-1+n)} g\left(z^{n}\right)+1 / t$, if $|t|=1$ and $2 \leqq n \in \omega$. Hence $g(z)(t)$ $=1 / t$. Since $g(z) \notin A$ we have $H^{2}(A, A) \neq\{0\}$.

Thus for three modules representing, perhaps, the most natural ones, we 
have examples of commutative semi-simple Banach algebras whose 2-dimensional cohomology groups do not all vanish.

3. Cohomology groups and the Wedderburn theorem. In this section we extend some of the results of $\S 6$ of [5] to commutative Banach algebras and bounded cochains. All our extensions are quite direct.

Definition 3.1. Suppose $A$ is a commutative Banach algebra. Then $\{B, \sigma, R\}$ is a normed extension of $A$ provided

(i) $B$ is a commutative Banach algebra;

(ii) $\sigma$ is a bounded homomorphism of $B$ onto $A$;

(iii) $R$ is the radical of $B$ and the kernel of $\sigma$;

(iv) there exists a bounded linear map $\rho$ of $A$ into $B$ such that $\sigma \rho(a)=a$ for all $a \in A$.

REMARK. If $B, \sigma, R$ satisfy (i), (ii), (iii) above, then (iv) is equivalent to (iv') $R$ has a Banach space complement in $B$.

REMARK. If $A$ is semi-simple, then every homomorphism of a Banach algebra $B$ into $A$ is bounded.

Definition 3.2. Let $A$ be a commutative Banach algebra. Two normed extensions $\{B, \sigma, R\}$ and $\left\{B^{\prime}, \sigma^{\prime}, R^{\prime}\right\}$ of $A$ are isomorphic provided there exists a bounded isomorphism $I$ of $B$ onto $B^{\prime}$ with $\sigma^{\prime} I=\sigma$.

Definition 3.3. Let $A$ be a commutative Banach algebra. The normed extension $\{B, \sigma, R\}$ is called singular provided $R^{2}=\{0\}$.

Definition 3.4. Let $A$ be a commutative Banach algebra. The normed extension $\{B, \sigma, R\}$ splits provided there exists a closed subalgebra $A^{\prime}$ of $B$ such that $B=A^{\prime} \oplus R$.

Definition 3.5. Let $A$ be a commutative Banach algebra and $(M, \cdot)$ a normed $A$-module. Then a bilinear map $f$ is a commuting $(2, M)$-cochain provided $f(a, b)=f(b, a)$ for all $a, b \in A$.

We note that if $f$ is a commuting $(2, M)$-cochain of $A$ and $f^{\prime}$ is cohomologous to $f$, then $f^{\prime}$ is also a commuting $(2, M)$-cochain of $A$. We can thus talk about commuting $(2, M)$-cohomology classes of $A$. These are the relevant ones here since the algebra itself is commutative.

Theorem 3.1. Suppose $A$ is a commutative semi-simple Banach algebra. If we identify corresponding cohomology classes over isomorphic normed A-modules, then there is a 1-1 correspondence between classes of isomorphic normed singular extensions of $A$ and bounded commuting 2-dimensional cohomology classes of $A$.

Proof. Consider the normed singular extension $\{B, \sigma, R\}$ of $A$. We shall associate with it a normed $A$-module $(R, \cdot)$ and a bounded $(2, R)$-cohomology class of $A$. As the definition of - take $a \cdot r=\rho(a) r$ for some $\rho$ satisfying (iv) of Definition 3.1. If $\rho_{1}$ also satisfies 3.1 (iv) and $a \circ r=\rho_{1}(a) r$, then $a \circ r=a \cdot r$ since $\rho_{1}(a)-\rho(a) \in R$ and $R^{2}=\{0\}$. Thus . is independent of the particular $\rho$ that is used. Furthermore $\|a \cdot r\| \leqq N\|a\|\|r\|$ where, in fact, $N$ may be taken to be inf $\|\rho\|$. We have therefore defined a normed $A$-module $(R, \cdot)$ which is completely determined by $\{B, \sigma, R\}$. 
The bounded bilinear map $f_{\rho}: f_{\rho}(a, b)=\rho(a) \rho(b)-\rho(a b)$ takes $A \times A$ into $R$ since $\sigma f_{\rho}(a, b)=0$ for all $a, b \in A$. One can verify that $f_{\rho}$ is a bounded commuting $(2, R)$-cocycle of $A$. If $\rho_{1}$ is another bounded linear map of $A$ into $B$ with $\sigma\left(\rho_{1}(a)\right)=a$, then it is shown in $\left[5\right.$, p. 66] that $f_{p}(a, b)=f_{\rho_{1}}(a, b)$ $+\delta\left(\rho-\rho_{1}\right)(a, b)$ and so $f_{\rho_{1}}$ is cohomologous to $f_{\rho \text {. }}$ Thus the bounded $(2, R)$ cohomology class $\tilde{f}_{p}$ of which $f_{p}$ is a member is uniquely determined in this manner by $\{B, \sigma, R\}$. We now show that if $\left\{B^{\prime}, \sigma^{\prime}, R^{\prime}\right\}$ is a normed singular extension of $A$ which is isomorphic under $I$ to $\{B, \sigma, R\}$, then the cohomology class determined by $\left\{B^{\prime}, \sigma^{\prime}, R^{\prime}\right\}$ in the manner described corresponds in a natural way to that determined by $\{B, \sigma, R\}$. First we observe that if $a \in A$, then

$$
\sigma^{\prime}\left(\rho^{\prime}(a)\right)=a=\sigma(\rho(a))=\sigma^{\prime} I(\rho(a))
$$

and so $\rho^{\prime}(a)-I \rho(a) \in R$. Hence

$$
a \cdot{ }^{\prime} I(r)=\rho^{\prime}(a) I(r)=(I \rho)(a) I(r)=I(\rho(a) r)=I(a \cdot r)
$$

showing that $I$ is also a bounded operator isomorphism between the two normed $A$-modules $(R, \cdot)$ and $\left(R^{\prime}, \cdot^{\prime}\right)$. As a result isomorphic normed singular extensions lead to isomorphic normed $A$-modules.

The map $I \rho$ is a bounded linear map of $A$ into $B^{\prime}$ with $\sigma^{\prime}(I \rho)(a)=a$. Hence $f_{I_{\rho}}$ is a bounded $\left(2, R^{\prime}\right)$-cocycle of $A$ which lies in the $\left(2, R^{\prime}\right)$-cohomology class of $f_{\rho^{\prime}}$. Since $f_{I_{\rho}}(a, b)=I f_{\rho}(a, b)$ for all $a, b \in A$, the bounded $\left(2, R^{\prime}\right)$-cohomology class $\tilde{f}_{\rho^{\prime}}$ determined by $\left\{B^{\prime}, \sigma^{\prime}, R^{\prime}\right\}$ is an isomorphic image by $I$ of the bounded $(2, R)$-cohomology class $f_{\rho}$ determined by $\{B, \sigma, R\}$.

Conversely suppose $(R, \cdot)$ is a normed $A$-module and $f$ is a bounded commuting $(2, R)$-cocycle of $A$. Let $B_{f}$ be the direct sum of $A$ and $R$ where for each $a \in A$ and $r \in R,\|(a, r)\|=(1+K)\|a\|+\|R\|\|r\|$ where $K=\max (\|f\|,\|R\|)$. If $(a, r)$ and $(b, s) \in B_{f}$ define a multiplication in $B_{f}$ by

$$
(a, r)(b, s)=(a b, a \cdot s+b \cdot r+f(a, b)) .
$$

Since $f$ is a bounded commuting $(2, R)$-cocycle of $A$ it can be shown that this multiplication is associative and commutative and that with these definitions and operations $B_{f}$ is a commutative Banach algebra.

The mapping $\sigma_{f}:(a, r) \rightarrow a$ is a bounded homomorphism of $B_{f}$ onto $A$ with the kernel of $\sigma_{f}, R_{f}$ equal to $\{(0, r) \mid r \in R\}$. Since $R_{f}^{2}=\{(0,0)\}$ and $B_{f} / R_{f}$ is semi-simple, $R_{f}$ is the radical of $B_{f}$. The map $\rho_{f}: a \rightarrow(a, 0)$ is bounded and linear and $\sigma_{f} \rho_{f}(a)=a$ for all $a \in A$. Therefore $\left\{B_{f}, \sigma_{f}, R_{f}\right\}$ is a normed singular extension of $A$. The bounded $\left(2, R_{f}\right)$-cocycle $f_{p g}$ derived from $\left\{B_{f}, \sigma_{f}, R_{f}\right\}$ is (to within a trivial correspondence) identical with $f$. Indeed $f_{\rho g}(a, b)=(0, f(a, b))$.

If $f$ and $f_{1}$ are corresponding bounded $(2, R)$ and $\left(2, R_{1}\right)$-cocycles over isomorphic normed $A$-modules $(R, \cdot)$ and $\left(R_{1}, \cdot 1\right)$, then it is easily shown that the corresponding normed singular extensions $\left\{B_{f}, \sigma_{f}, R_{f}\right\}$ and $\left\{B_{f_{1}}, \sigma_{f_{1}}, R_{f_{1}}\right\}$ are isomorphic. 
Finally let $f^{\prime}$ be a bounded $(2, R)$-cocycle of $A$ which is cohomologous to $f$, with $g$ bounded linear and $f=f^{\prime}+\delta g$. Consider the map $J:(a, r) \rightarrow(a, r+g(a))$ which takes $B_{f}$ onto $B_{f^{\prime}}$. Since $f=f^{\prime}+\delta g$, we can verify that $J((a, r)(b, s))$ $=J(a, r) J(b, s)$ showing that $J$ is a bounded isomorphism. Moreover $\sigma_{f^{\prime}}(J((a, r)))=\sigma_{f^{\prime}}((a, r+g(a)))=a=\sigma_{f}((a, r))$, so that $\sigma_{f^{\prime}} J=\sigma_{f}$. Hence the normed singular extensions $\left\{B_{f}, \sigma_{f}, R_{f}\right\}$ and $\left\{B_{f^{\prime}}, \sigma_{f^{\prime}}, R_{f^{\prime}}\right\}$ are isomorphic and all is proved.

Theorem 3.2. Suppose $\{B, \sigma, R\}$ is a normed singular extension of the commutative semi-simple Banach algebra $A$. Then the following are equivalent:

(i) $\{B, \sigma, R\}$ splits;

(ii) The $(2, R)$-cocycle $f_{\rho}$ is cohomologous to 0 .

Proof. If (i) holds, $\sigma$ is easily seen to give an isomorphism of $A^{\prime}$ onto $A$. Let $\rho$ be the inverse of this isomorphism. Since $\rho(a b)=\rho(a) \rho(b)$ we have $f_{\rho}=0$ and thus (ii) holds.

Conversely if $f_{\rho}$ is cohomologous to 0 , then $\{B, \sigma, R\}$ is isomorphic to $\left\{B_{0}, \sigma_{0}, R_{0}\right\}$. Here $B_{0}=B_{f}$ with $f=0$. Since $B_{0}=A \oplus R$ and the decomposition preserves multiplication in $B$, we have the necessary direct sum.

Corollary 3.3. Let $A$ be a commutative semi-simple Banach algebra. A necessary and sufficient condition that every normed singular extension $\{B, \sigma, R\}$ of $A$ split is that for every normed $A$-module $(M, \cdot)$, each bounded commuting $(2, M)$-cocycle is a bounded $(2, M)$-coboundary.

If $H^{2}(A, M)=\{0\}$ for all normed $A$-modules $(M, \cdot)$, then every normed singular extension $\{B, \sigma, R\}$ of $A$ splits.

Definition 3.6. Let $A$ be a commutative Banach algebra. $\{B, \sigma, R\}$ is an $(N)$-extension of $A$ provided it is a normed extension, $R$ is nilpotent and every even power of $R$ has a Banach space complement in $B$.

REMARK. Every normed extension $\{B, \sigma, R\}$ with $R$ finite dimensional is an $(N)$-extension.

TheOREM 3.4. Suppose $A$ is a commutative semi-simple Banach algebra such that every normed singular extension splits. Then every $(N)$-extension splits.

Proof. Let the order of $R$ be the smallest integer $n$ for which $R^{n}=\{0\}$. The proof is by induction on the order of $R$. If order $R=1$, then $R=\{0\}$ and $B$ is semi-simple.

Suppose the theorem holds when order $R<n$. Precisely we assume that if $\left\{B_{1}, \sigma_{1}, R_{1}\right\}$ is an $(N)$-extension of $A$ and $R^{n-1}=\{0\}$, then $\left\{B_{1}, \sigma_{1}, R_{1}\right\}$ splits.

Let $\{B, \sigma, R\}$ be an $(N)$-extension with $R$ having order $n$. For each $k=1, \cdots,[(n+1) / 2]$, let $S_{k}$ be a closed subspace of $B$ with $B=S_{k} \oplus R^{2 k^{-}}$ and $S$ be the Banach space complement of $R . R^{2^{-}}$and $R^{2 k^{-}}$will denote, respectively, the Banach space closure of $R^{2}$ and $R^{2 k}$. 
Consider $B / R^{2^{-}}$and the map $\bar{\sigma}$ of $B / R^{2^{-}}$onto $A$ defined by $\bar{\sigma}(\bar{b})=\sigma(b)$ for $\bar{b} \in B / R^{2^{-}}$. Then $\bar{\sigma}^{-1}(0)=R / R^{2^{-}}$and since $R / R^{2^{-}}$has a Banach space complement in $B / R^{2^{-}},\left\{B / R^{2^{-}}, \bar{\sigma}, R / R^{2^{-}}\right\}$is a normed singular extension. Thus by hypothesis there is a closed subalgebra $\bar{A}$ of $B / R^{2^{-}}$such that $B / R^{2^{-}}$ $=\bar{A} \oplus R / R^{2^{-}}$with $\bar{A}$ isomorphic to $A$.

Let $C=\{c \in B \mid \bar{c} \in \bar{A}\}$. Then $C$ is a closed subalgebra of $B$ and the map $\tau: \tau(c)=\bar{c}$ is a homomorphism of $C$ onto $A$ (identifying $A$ and $\bar{A}$ ). The kernel of $\tau$ is $R^{2^{-}}$and since $C=\left(C \cap S_{k}\right) \oplus R^{2 k^{-}},\left\{C, \tau, R^{2^{-}}\right\}$is an $(N)$-extension of $A$ with order $R^{2^{-}}<n$. Hence there exists a closed subalgebra $A^{\prime}$ of $C$ (and thus of $B$ ) such that $C=A^{\prime} \oplus R^{2^{-}}$.

Since $B / R^{2^{-}}=\bar{A} \oplus R / R^{2^{-}}$from $\bar{A}=C / R^{2^{-}}$and $C=A^{\prime} \oplus R^{2^{-}}$it follows that every $b$ in $B$ is expressible as a sum $a_{b}^{\prime}+r_{b}$ where $a_{b}^{\prime} \in A^{\prime}$ and $r_{b} \in R$. If $a^{\prime} \in A^{\prime} \cap R$, then $\left(a^{\prime}\right)^{n}=0$. But $A^{\prime}$ being semi-simple implies $a^{\prime}=0$ and hence $B=A^{\prime} \oplus R$, which is what we set out to prove.

In an analogous, though simpler, manner one can prove

TheOREM 3.5. Let $A$ be a commutative semi-simple Banach algebra. If for every normed singular extension $\{B, \sigma, R\}$ of $A$ with $R$ finite dimensional we have $B=A^{\prime} \oplus R$ for some closed subalgebra $A^{\prime}$ of $B$, then such a decomposition holds for all normed extensions $\{B, \sigma, R\}$ of $A$ where $R$ is finite dimensional.

A consequence of Theorem 3.5 is that if $H^{2}(A, M)=\{0\}$ for all normed $A$-modules $(M, \cdot)$ with $M$ finite dimensional, then if $B$ is a commutative $\mathrm{Ba}$ nach algebra with finite dimensional radical, and $B / R=A$, then there exists a closed subalgebra $A^{\prime}$ of $B$ with $B=A^{\prime} \oplus R$.

Theorem 3.6. Let $A$ be a commutative Banach algebra. Then the following are equivalent.

(i) If $\{B, \sigma, R\}$ is a normed singular extension of $A$ and $B=A_{1} \oplus R$ $=A_{2} \oplus R$ where $A_{1}$ and $A_{2}$ are closed subalgebras of $B$, then $A_{1}=A_{2}$.

(ii) $H^{1}(A, M)=\{0\}$ for all normed $A$-modules $(M, \cdot)$.

Proof. We show first that if $H^{1}(A, M) \neq\{0\}$ for some normed $A$-module $(M, \cdot)$, then (i) fails. To this end let $d$ be a bounded derivation of $A$ into $M$ and let $B=\{(a, m) \mid a \in A$ and $m \in M\}$ where multiplication in $B$ is defined by $(a, m)\left(a^{\prime}, m^{\prime}\right)=\left(a a^{\prime}, a^{\prime} \cdot m+a \cdot m^{\prime}\right)$ for all $(a, m),\left(a^{\prime}, m^{\prime}\right) \in B$. If $\sigma$ is the map taking $B$ onto $A$ defined by $\sigma((a, m))=a$ for all $(a, m) \in B$ and if $R=\{(0, m) \in B \mid m \in M\}$, then $\{B, \sigma, R\}$ is a normed singular extension of $A$. Clearly if $A_{1}=\{(a, 0) \mid a \in A\}$, then $B=A_{1} \oplus R$. Also $A_{2}=\{(a, d(a)) \mid a \in A\}$ is a closed subalgebra of $B$ with $B=A_{2} \oplus R$. Hence this decomposition is not unique.

Conversely assume $H^{1}(A, M)=\{0\}$ for all normed $A$-modules $(M, \cdot)$. Suppose $\{B, \sigma, R\}$ is a normed singular extension of $A$ and $B=A_{1} \oplus R$ $=A_{2} \oplus R$. Let $I$ be an isomorphism of $A$ onto $A_{1}$ and define a normed $A$ module $(R, \cdot)$ by $a \cdot r=I(a) r$. Since $A_{1} \subset B$ we can write each $a_{1} \in A_{1}$ uniquely 
as $a_{1}=h\left(a_{1}\right)+r\left(a_{1}\right)$ where $h\left(a_{1}\right) \in A_{2}$ and $r\left(a_{1}\right) \in R$. Clearly the map $a_{1} \rightarrow h\left(a_{1}\right)$ is an isomorphism of $A_{1}$ into $A_{2}$. Also one can verify that $a \rightarrow r(I(a))$ is a bounded derivation of $A$ into $(R, \cdot)$. Since $H^{1}(A, R)=\{0\}, r(I(a))=0$ and so $a_{1}=h\left(a_{1}\right)$. Thus $A_{1}$ is a subalgebra of $A_{2}$. Similarly $A_{2}$ is a subalgebra of $A_{1}$. Consequently $A_{1}=A_{2}$ as needed.

An induction argument can be used to show that this theorem remains valid if $\{B, \sigma, R\}$ is any normed extension of $A$ with $R$ nilpotent.

4. Cohomology groups of $C(X) .\left(^{3}\right)$ The main object of this section is to prove that the cohomology groups $H^{1}(C(X), M)$ and $H^{2}(C(X), M)$ are trivial for all normed $C(X)$-modules $(M, \cdot)$.

Theorem 4.1 of [2] asserts that if $X$ is a compact Hausdorff space, if $B$ is a commutative Banach algebra with radical $R$, if $B / R=C(X)$, and if there exists a closed subalgebra $A$ of $B$ with $B=A \oplus R$, then $A$ is unique. Using this and Theorem 3.6, we have the following.

Theorem 4.1. Let $X$ be a compact Hausdorff space and $(M, \cdot)$ a normed $C(X)$-module. Then $H^{1}(C(X), M)=\{0\}$.

In Corollary 2.2 of [10], Singer and Wermer have proved that there are no nonzero derivations of $C(X)$ into any semi-simple commutative extension $B$. Theorem 4.1 shows that there are no nonzero bounded derivations of $C(X)$ into any commutative extension of $C(X)$.

In order to prove that $H^{2}(C(X), M)=\{0\}$ for all normed $C(X)$-modules $(M, \cdot)$ we shall use some results on extensions of bounded multilinear maps. First let $P$ be an arbitrary Banach space. For each bounded linear functional $h$ on $P$ we write $(x, h)$ for the result of the operator $h$ on the element $x \in P$. $P^{*}$ will denote the linear space of bounded linear functionals on $P$ and setting $\|h\|=\sup \{|(x, h)| \mid x \in P$ and $\|x\|=1\}, P^{*}$ is a Banach space. There exists an isometric isomorphism $\phi$ of $P$ into $P^{* *}$ such that for all $h \in P^{*},(x, h)=(h, \phi(x))$. When there is no possibility of confusion the same symbol $x$ will be used for both the element $x \in P$ and its image $\phi(x)$ in $P^{* *}$. Further we shall write $\phi(P)$ as $P$. In this sense, then, a Banach space $P$ is reflexive if, and only if, $P=P^{* *}$.

The $w^{*}$-topology in $P^{*}$ is that topology generated by sets of the form $N\left(h_{0}, p_{1}, \cdots, p_{n} ; \epsilon\right)=\left\{h \in P^{*}||\left(p_{i}, h\right)-\left(p_{i}, h_{0}\right) \mid<\epsilon, i=1, \cdots, n\right\}$ where $h_{0} \in P^{*}, p_{1}, \cdots, p_{n} \in P$ and $\epsilon>0$. A net $\left\{h_{n}\right\}_{n \in N}$ in $P^{*}$ is $w^{*}$-convergent to $h \in P^{*}$ provided $\left(y, h_{n}\right) \rightarrow(y, h)$ for all $y \in P$. Since $P$ is $w^{*}$-dense in $P^{* *}$, if $\bar{x} \in P^{* *}$, there exists a net $\left\{x_{n}\right\}_{n \in N}$ in $P$ which is $w^{*}$-convergent to $\bar{x}$.

Let $U$ be a bounded linear map of a Banach space $P$ into a Banach space $Q$. Then the adjoint map $U^{*}: Q^{*} \rightarrow P^{*}$ is defined by $\left(p, U^{*} q^{*}\right)=\left(U p, q^{*}\right)$ for all $p \in P$ and $q^{*} \in Q^{*}$. $U^{* *}: P^{* *} \rightarrow Q^{* *}$ is defined by $U^{* *}=\left(U^{*}\right)^{*}$. The properties of $U^{*}$ and $U^{* *}$ are well known. In particular if $p \in P, U^{* *}(p)=U(p)$.

(3) The author thanks the referee for indicating shortcuts in the original proofs of Theorems 4.1 and 4.2. 
Now suppose that $P, Q$ and $R$ are Banach spaces and $f$ is a bounded bilinear map of $P \times Q$ into $R$. Then in [1] Arens defined an extension of $f$ to a map $f^{* * *}: P^{* *} \times Q^{* *} \rightarrow R^{* *}$. This map, the adjoint of $f$, was defined in the following way. First define $f^{*}: R^{*} \times P \rightarrow Q^{*}$ by $\left(q, f^{*}\left(r^{*}, p\right)=\left(f(p, q), r^{*}\right)\right.$ for all $p \in P, q \in Q$ and $r^{*} \in R^{*}$. Then let $f^{* *}=\left(f^{*}\right)^{*}$ and $f^{* * *}=\left(f^{* *}\right)^{*}$. $f^{* * *}$ has the property that $f^{* * *}(p, q)=f(p, q)$ for all $p \in P$ and $q \in Q$. Theorem 3.2 of $[1]$ asserts that for fixed $\bar{q} \in Q^{* *}, \bar{p} \rightarrow f^{* * *}(\bar{p}, \bar{q})$ is $w^{*}$-continuous and also for fixed $p \in P, \bar{q} \rightarrow f^{* * *}(p, \bar{q})$ is $w^{*}$-continuous. In general, though, $\bar{q} \rightarrow f^{* * *}(\bar{p}, \bar{q})$ is not $w^{*}$-continuous. Adopting the terminology of [1], we shall call the bounded bilinear map $f$ regular if, and only if, $\bar{q} \rightarrow f^{* * *}(\bar{p}, \bar{q})$ is $w^{*}$-continuous for fixed $\bar{\rho} \in P^{* *}$. Theorem 4.2 will show that when $P=Q=C(X)$ each bounded bilinear map on $C(X) \times C(X)$ is regular.

If $A$ is a Banach algebra, if $P=Q=R=A$, and if $S: A \times A \rightarrow A$ is defined by $S(x, y)=x y$, then $S^{* * *}$ is an associative multiplication making $A^{* *}$ into a Banach algebra which, however, need not be commutative even if $A$ is commutative. If $A$ is commutative, then regularity of $S$ means that $A^{* *}$ is commutative. In case $X$ is a compact Hausdorff space the algebra $C(X)^{* *}$ is a commutative Banach algebra and is, in fact, isometrically isomorphic to a $C(Y)$ for some extremely disconnected compact Hausdorff space $Y$.

If $A$ is Banach algebra, $(M, \cdot)$ is a normed $A$-module, and if $T: A \times M \rightarrow M$ is defined by $T(a, m)=a \cdot m$, then the extension $T^{* * *}: A^{* *} \times M^{* *} \rightarrow M^{* *}$ defines a normed $A^{* *}$-module $\left(M^{* *}, \cdot\right)$ using the adjoint multiplication defined in the last paragraph. There is no confusion in using the same symbol $\cdot$ for both module operations since on $A \times M, T^{* * *}=T$. It is conceivable that the adjoint of a bounded $(k, M)$-cocycle is not a bounded $\left(k, M^{* *}\right)$-cocycle of $A^{* *}$. However, we shall see that if $A=C(X), X$ compact Hausdorff, then the adjoint of a bounded $(k, M)$-cocycle of $C(X)$ is a bounded $\left(k, M^{* *}\right)$ cocycle of $C(X)^{* *}$.

THEOREM 4.2. If $X$ is a compact Hausdorff space and $R$ is a Banach space, then every bounded bilinear map $f$ of $C(X) \times C(X)$ into $R$ is regular.

Proof. Assume first that $R$ is the complex number field. We must show that for fixed $\bar{x} \in C(X)^{* *}$, the map $\bar{y} \rightarrow f^{* *}(\bar{x}, \bar{y})$ is $w^{*}$-continuous on $C(X)^{* *}$. To this end define the bounded linear map $T: C(X) \rightarrow C(X)^{*}$ by $(T x)(y)$ $=f(x, y)$ for all $x, y \in C(X)$. Then it is easily seen that for $\bar{x}, \bar{y} \in C(X)^{* *}$, $\left(\bar{y}, T^{* *} \bar{x}\right)=f^{* * *}(\bar{x}, \bar{y})$ where in considering $f^{* * *}(\bar{x}, \bar{y})$ we make the natural identification of the complex number field with its double dual space. Since $C(X)^{*}$ is weakly complete [3, IV. 13.22], Theorem VI. 7.6 of [3] implies that the operator $T$ is weakly compact. Hence by VI. 4.2 of [3], $T^{* *} \operatorname{maps} C(X)^{* *}$ into the natural embedding $\phi\left(C(X)^{*}\right)$ of $C(X)^{*}$ in $C(X)^{* * *}$, i.e., $T^{* *} \bar{x} \in C(X)^{*}$ for all $\bar{x} \in C(X)^{* *}$. Thus if $\left\{\bar{y}_{n}\right\}_{n \in N}$ is a net in $C(X)^{* *}$ and $\bar{y}_{n} \rightarrow \bar{y}$ in the $w^{*}$ topology of $C(X)^{* *}$, then for fixed $\bar{x} \in C(X)^{* *}$, we have $f^{* * *}\left(\bar{x}, \bar{y}_{n}\right)=\left(T^{* *} \bar{x}, \bar{y}_{n}\right)$ $\rightarrow\left(T^{* *} \bar{x}, \bar{y}\right)=f^{* * *}(\bar{x}, \bar{y})$, i.e. for fixed $\bar{x} \in C(X)^{* *}$, the map $\bar{y} \rightarrow f^{* * *}(\bar{x}, \bar{y})$ is $w^{*}$-continuous. 
For arbitrary $R$ the result follows from this by noting that, for each $h \in R^{*}$, the map $F_{h}:(x, y) \rightarrow(f(x, y), h)$ is regular and that $F_{h}^{* * *}(\bar{x}, \bar{y})$ $=\left(h, f^{* * *}(\bar{x}, \bar{y})\right)$ for all $\bar{x}, \bar{y} \in C(X)^{* *}$, again making the proper identification of the complex numbers with its second conjugate space.

It follows from this theorem that if $f$ is a bounded commuting $(2, M)$ cochain of $C(X)$, then $f^{* * *}$ is a bounded commuting $\left(2, M^{* *}\right)$-cochain of $C(X)^{* *}$. In particular this shows that multiplication in $C(X)^{* *}$ is commutative.

In a similar way to [1] one can define the adjoint $f^{(k+1) *}$ of a bounded $k$-linear map $f$. Thus if $f$ is a $k$-linear of $P_{1} \times \cdots \times P_{k}$ into $Q$, let $f^{1 *}: Q^{*}$ $\times P_{1} \times \cdots \times P_{k-1} \rightarrow P_{k}^{*}$ be defined by $\left(p_{k}, f^{1 *}\left(q^{*}, p_{1}, \cdots, p_{k-1}\right)\right)$ $=\left(f\left(p_{1}, \cdots, p_{k}\right), q^{*}\right)$ for $p_{i} \in P_{i}$ and $q^{*} \in Q^{*}$. Let $f^{(i) *}=\left(f^{(i-1) *}\right)^{*}$. The following theorem can be proved with this definition.

TheOREM 4.3. Let $P$ and $Q$ be Banach spaces such that each bounded bilinear operator from $P \times P$ into $Q$ is regular. If $k \in \omega$ and $f$ is a bounded k-linear map of $P^{k}$ into $Q$ with adjoint $f^{(k+1) *}$, then $f^{(k+1) *}$ is $w^{*}$-continuous in each variable holding the other variables fixed.

TheOREM 4.4. Let $X$ be a compact Hausdorff space and $(M, \cdot)$ a normed $C(X)$-module. If $f$ is a bounded $(2, M)$-cocycle of $C(X)$, then $f^{* * *}$ is a bounded $\left(2, M^{* *}\right)$-cocycle of $C(X)^{* *}$.

Proof. Suppose $\bar{x}, \bar{y}, \bar{z} \in C(X)^{* *}$ and $\left\{x_{k}\right\}_{k \in K},\left\{y_{n}\right\}_{n \in N}$ and $\left\{z_{p}\right\}_{p \in P}$ are nets in $C(X)$ which approach $\bar{x}, \bar{y}$ and $\bar{z}$, respectively, in the $w^{*}$-topology of $C(X)^{* *}$. Since $f$ is a $(2, M)$-cocycle, we have

$$
x_{k} \cdot f\left(y_{n}, z_{p}\right)-f\left(x_{k} y_{n}, z_{p}\right)+f\left(x_{k}, y_{n} z_{p}\right)-z_{p} \cdot f\left(x_{k}, y_{n}\right)=0 \text {. }
$$

In general,

and

$$
\begin{aligned}
w^{*}-\lim & \lim _{n} \lim _{p} x_{k} \cdot f\left(y_{n}, z_{p}\right)=\bar{x} \cdot f^{* * *}(\bar{y}, \bar{z}), \\
w^{*}-\lim _{k} \lim _{n} \lim _{p} f\left(x_{k} y_{n}, z_{p}\right) & =f^{* * *}(\bar{x} \bar{y}, \bar{z}),
\end{aligned}
$$

$$
w^{*}-\lim _{k} \lim _{n} \lim _{p} f\left(x_{k}, y_{n} \bar{z}_{p}\right)=f^{* * *}(\bar{x}, \bar{y} \bar{z})
$$

Also

$$
\begin{aligned}
w^{*}-\lim _{z} \lim _{n} \lim _{\nu} z_{p} \cdot f\left(x_{k}, y_{n}\right) & =w^{*}-\lim _{k} \lim _{n} \bar{z} \cdot f^{* * *}\left(x_{k}, y_{n}\right) \\
& =w^{*}-\lim _{k} \bar{z} \cdot f^{* * *}\left(x_{k}, \bar{y}\right)=\bar{z} \cdot f^{* * *}(\bar{x}, \bar{y}) .
\end{aligned}
$$

The last two equalities follow from Theorem 4.3. Thus

$$
\bar{x} \cdot f^{* * *}(\bar{y}, \bar{z})-f^{* * *}(\bar{x} \bar{y}, \bar{z})+f^{* * *}(\bar{x}, \bar{y} \bar{z})-\bar{z} \cdot f^{* * *}(\bar{x}, \bar{y})=0
$$

and so $f^{* * *}$ is a bounded $\left(2, M^{* *}\right)$-cocycle of $C(X)^{* *}$. 
REMARK. In a similar way the adjoint of a $(k, M)$-cocycle of $C(X)$ is a $\left(k, M^{* *}\right)$-cocycle of $C(X)^{* *}$ for all $k \in \omega$.

In Theorem 4.1 it was shown that $H^{1}(C(X), M)=\{0\}$ for all normed $C(X)$-modules $(M, \cdot)$. Another proof of this is the following. Let $d$ be a bounded derivation of $C(X)$ into $M$. Then $d^{* *}$ is a bounded derivation of $C(X)^{* *}$ into $M^{* *}$. For a characteristic function $E$ of a Borel subset of $X$ we have $d^{* *}(E)=d^{* *}\left(E^{2}\right)=2 E \cdot d^{* *}(E)$ and so $E \cdot d^{* *}(E)=2 E \cdot d^{* *}(E)=0$. Thus $d^{* *}(E)=0$. Since every $x \in C(X)$ may be approximated by finite linear combinations of these characteristic functions, we obtain $d^{* *}(x)=0$ and hence $d(x)=0$ for all $x \in C(X)$.

The next step is to show that $H^{2}(C(X), M)=\{0\}$ for all normed unital $C(X)$-modules $(M, \cdot)$. This could be done directly, and in the case $X$ is compact metric such a proof may be found in [6]. However a simpler proof again follows by using a result of Bade and Curtis [2]. Recall that they have shown that if $Y$ is an extremely disconnected compact Hausdorff space and $B$ is a commutative Banach algebra with radical $R$ satisfying $B / R=C(Y)$, then there exists a closed subalgebra $A^{\prime}$ of $B$ with $B=A^{\prime} \oplus R$. From Corollary 3.3 it then follows that for every normed $C(Y)$-module $(M, \cdot)$, each bounded commuting $(2, M)$-cocycle of $C(Y)$ is a bounded $(2, M)$-coboundary. We now use this result to show that for an arbitrary compact Hausdorff space $X$ and an arbitrary normed $C(X)$-module $(M, \cdot)$, every bounded commuting $(2, M)$-cocycle of $C(X)$ is a bounded $(2, M)$-coboundary.

In the course of the proof we shall need the following lemma which was alluded to in $\$ 2$.

Lemma 4.5. Suppose $f$ is a bilinear map of an algebra $A$ into an $A$-module $(M, \cdot)$. If $g$ is a linear map of $A$ into $M$ and $\delta g=f$, then for $2 \leqq n \in \omega$,

$$
n u^{n-1} \cdot g(u)-g\left(u^{n}\right)=\sum_{i=0}^{n-2} u^{i} \cdot f\left(u, u^{n-i-1}\right)
$$

Proof. For $n=2$ it follows directly that $2 u \cdot g(u)-g\left(u^{2}\right)=f(u, u)$. Assume statement $(4.5)$ is true for $k-1$, i.e.

$$
(k-1) u^{k-2} \cdot g(u)-g\left(u^{k-1}\right)=\sum_{i=0}^{k-3} u^{i} \cdot f\left(u, u^{k-i-2}\right) .
$$

From $\delta g\left(u, u^{k-1}\right)=f\left(u, u^{k-1}\right)$ we obtain

$$
u \cdot g\left(u^{k-1}\right)-g\left(u^{k}\right)+u^{k-1} \cdot g(u)=f\left(u, u^{k-1}\right) .
$$

Combining (4.6) and (4.7) we find

$$
-g\left(u^{k}\right)+(k-1) u^{k-1} \cdot g(u)-u \cdot \sum_{i=0}^{k-3} u^{i} \cdot f\left(u, u^{k-i-2}\right)+u^{k-1} \cdot g(u)=f\left(u, u^{k-1}\right)
$$

and so 


$$
k u^{k-1} \cdot g(u)-g\left(u^{k}\right)=\sum_{i=0}^{k-3} u^{i+1} \cdot f\left(u, u^{k-i-2}\right)+f\left(u, u^{k-1}\right)=\sum_{i=0}^{k-2} u^{i} \cdot f\left(u, u^{k-i-1}\right)
$$

and all is done.

TheOREM 4.6. Let $X$ be a compact Hausdorff space and $(M, \cdot)$ a unital normed $C(X)$-module. Suppose $f$ is a bounded commuting $(2, M)$-cocycle of $C(X)$. Then there exists a bounded $(1, M)$-cochain $g$ of $C(X)$ with $f=\delta g$.

Proof. Let $f^{* * *}$ be the adjoint of $f$. From Theorem $4.4, f^{* * *}$ is a $\left(2, M^{* *}\right)$ cocycle of $C(X)^{* *}$. Since $C(X)^{* *}=C(Y)$ for some extremely disconnected $Y$, there exists a bounded $\left(1, M^{* *}\right)$-cochain $g$ of $C(X)^{* *}$ with $\delta g=f^{* * *}$. Thus for $x, y \in C(X)$,

$$
x \cdot g(y)-g(x y)+y \cdot g(x)=f^{* * *}(x, y) \in M
$$

where we again identify $M$ with its natural embedding in $M^{* *}$ and $C(X)$ with its natural embedding in $C(X)^{* *}$.

By Lemma 4.5,

$$
n u^{n-1} \cdot g(u)-\sum_{i=0}^{n-2} u^{i} \cdot f\left(u, u^{n-i-1}\right)=g\left(u^{n}\right) .
$$

If $u \in C(X)$ and $|u(t)|=1$ for all $t \in X$, then

$$
g(u)-\frac{1}{n} \sum_{i=0}^{n-2} u^{i-n+1} \cdot f\left(u, u^{n-i-1}\right)=\frac{u^{1-n}}{n} \cdot g\left(u^{n}\right)
$$

Thus

$$
\left\|g(u)-\frac{1}{n} \sum_{i=0}^{n-2} u^{i-n+1} \cdot f\left(u, u^{n-i-1}\right)\right\| \leqq \frac{1}{n}\|g\|\|M\|
$$

Now

$$
\frac{1}{n} \sum_{i=0}^{n-2} u^{i-n+1} \cdot f\left(u, u^{n-i-1}\right) \in M \quad \text { and } \quad \frac{1}{n} \sum_{i=0}^{n-2} u^{i-n+1} \cdot f\left(u, u^{n-i-1}\right) \rightarrow g(u)
$$

in the norm of $C(X)^{* *}$. Since $M$ is closed in the norm topology as a subspace of $M^{* *}$ it follows that $g(u) \in M$ when $u \in C(X)$ and $|u(t)|=1$ for all $t \in X$. But every continuous function $w$ may be expressed as a linear combination of four functions of this form. Indeed

$$
\begin{aligned}
w= & \frac{\|w\|}{2 i}\left[\operatorname { e x p } \left(i \sin ^{-1}\left(w_{1} /\|w\|\right)-\exp \left(-i \sin ^{-1}\left(w_{1} /\|w\|\right)\right.\right.\right. \\
& \left.+i \exp \left(i \sin ^{-1}\left(w_{2} /\|w\|\right)\right)-i \exp \left(-i \sin ^{-1}\left(w_{2} /\|w\|\right)\right)\right],
\end{aligned}
$$

where $w_{1}$ is the real part of $w$ and $w_{2}$ is the imaginary part of $w$. Since each of 
the four terms in the bracket is a continuous function with absolute value equal to 1 we have that $g(w) \in M$ for all $w \in C(X)$ as needed. Thus $g$ is a bounded $(1, M)$-cochain of $C(X)$ with $\delta g=f$.

TheOREM 4.7. Let $X$ be a compact Hausdorff space and $(M, \cdot)$ a unital normed $C(X)$-module. Then $H^{2}(C(X), M)=\{0\}$.

Proof. We shall show first that if $X$ is extremely disconnected and $f$ is a bounded $(2, M)$-cocycle of $C(X)$, then $f$ is commuting. To this end let $E, E^{\prime}$ and $E^{\prime \prime}$ be mutually orthogonal idempotents in $C(X)$ (i.e., $E E^{\prime}=E E^{\prime \prime}=E^{\prime} E^{\prime \prime}$ $=0)$. Then using the fact that $f$ is a $(2, M)$-cocycle it follows that

$$
\begin{aligned}
& E \cdot f\left(E^{\prime}, E\right)=f\left(E E^{\prime}, E\right)+f\left(E, E^{\prime} E\right)-E \cdot f\left(E, E^{\prime}\right)=0, \\
& E \cdot f\left(E^{\prime}, E\right)=E \cdot f\left(E, E^{\prime}\right) .
\end{aligned}
$$

In the same way $E^{\prime} \cdot f\left(E^{\prime}, E\right)=E^{\prime} \cdot f\left(E, E^{\prime}\right)$.

Furthermore

$$
E^{\prime \prime} \cdot f\left(E, E^{\prime}\right)-f\left(E^{\prime \prime} E, E^{\prime}\right)+f\left(E^{\prime \prime}, E E^{\prime}\right)-E^{\prime} \cdot f\left(E^{\prime \prime}, E\right)=0,
$$

and so $E^{\prime \prime} \cdot f\left(E, E^{\prime}\right)=0$. Similarly $E^{\prime \prime} \cdot f\left(E^{\prime}, E\right)=0$.

Now consider $E, E^{\prime}$ and $E^{\prime \prime}=1-E-E^{\prime}$ where 1 is the multiplicative identity in $C(X)$. Then $f\left(E, E^{\prime}\right)=\left(E+E^{\prime}+E^{\prime \prime}\right) \cdot f\left(E, E^{\prime}\right)=\left(E+E^{\prime}+E^{\prime \prime}\right)$ $\cdot f\left(E^{\prime}, E\right)=f\left(E^{\prime}, E\right)$. Hence if $E$ and $E^{\prime}$ are orthogonal idempotents, then $f\left(E, E^{\prime}\right)=f\left(E^{\prime}, E\right)$.

Let $x, y \in C(X)$. Then to each $\epsilon>0$ there exist mutually orthogonal idempotents $\left\{E_{i}\right\}_{i=1}^{n}$ and constants $x_{i}, y_{i}, i=1, \cdots, n$ with $\left\|x-\sum_{i=1}^{n} x_{i} E_{i}\right\|<\epsilon$ and $\left\|y-\sum_{i=1}^{n} y_{i} E_{i}\right\|<\epsilon$. Hence $\left\|f(x, y)-\sum_{t=1}^{n} \sum_{j=1}^{n} x_{i} y_{i} f\left(E_{i}, E_{j}\right)\right\|$ $\leqq\left\|f\left(x-\sum_{i=1}^{n} x_{i} E_{i}, y\right)\right\|+\left\|f\left(\sum_{i=1}^{n} x_{i} E_{i}, y-\sum_{j=1}^{n} y_{j} E_{j}\right)\right\| \leqq[\|y\|+\|x\|] \epsilon$. Also $\left\|f(y, x)-\sum_{j=1}^{n} \sum_{i=1}^{n} x_{i} y_{j} f\left(E_{j}, E_{i}\right)\right\| \leqq[\|y\|+\|x\|] \epsilon$. Since $f\left(E_{i}, E_{j}\right)=f\left(E_{j}, E_{i}\right)$, $i, j=1, \cdots, n$, it follows that $\|f(x, y)-f(y, x)\| \leqq 2 \epsilon[\|x\|+\|y\|]$. Thus $f(x, y)=f(y, x)$ in the case $X$ is extremely disconnected.

In the general case, if $f$ is a bounded $(2, M)$-cocycle of $C(X)$, then $f^{* * *}$ is a bounded $\left(2, M^{* *}\right)$-cocycle of $C(X)^{* *}$. Since $C(X)^{* *}$ is $C(Y)$ where $Y$ is extremely disconnected, $f^{* * *}$ is a commuting $\left(2, M^{* *}\right)$-cocycle and thus $f$ is a commuting $(2, M)$-cocycle of $C(X)$ since $f^{* * *}$ restricted to $C(X) \times C(X)$ equals $f$.

Thus by Theorem 4.6, $H^{2}(C(X), M)=\{0\}$ since that theorem says that every bounded commuting $(2, M)$-cocycle is a bounded $(2, M)$-coboundary.

Corollary 4.8. Let $X$ be a compact Hausdorff space. Then for all normed $C(X)$-modules $(M, \cdot), H^{2}(C(X), M)=\{0\}$.

This corollary follows from Theorem 4.7 and the remark after Theorem 1.5 .

If $X$ is locally compact but not compact then $C(X)$ denotes the algebra, with uniform norm, of complex-valued continuous functions on $X$ which 
vanish at the point of infinity. This commutative Banach algebra has no identity. If we adjoint a unit to $C(X)$, then the new algebra is isomorphic to $C\left(X^{\prime}\right)$ where $X^{\prime}$ is the one point compactification of $X$.

If $X$ is locally compact and $(M, \cdot)$ is a normed $C(X)$-module, then $(M, \cdot)$ can be extended in the canonical manner to be a unital normed $C\left(X^{\prime}\right)$ module. Thus using Theorem 1.6 we have the following.

CoROLlARY 4.9. Let $X$ be a locally compact Hausdorff space and $(M, \cdot)$ a normed $C(X)$-module. Then $H^{1}(C(X), M)=\{0\}$ and $H^{2}(C(X), M)=\{0\}$.

THEOREM 4.10. Let $X$ be a locally compact Hausdorff space.

(1) Let $B$ be a commutative Banach algebra with radical $R$. If $R$ is nilpotent and $R$ and the closure of every even power of $R$ have a Banach space complement in $B$ and if $B / R=C(X)$, then there exists a unique closed subalgebra $A^{\prime}$ of $B$ with $B=A^{\prime} \oplus R$.

(2) Let $B$ be a commutative Banach algebra with finite dimensional radical. If $B / R=C(X)$, then there exists a unique closed subalgebra $A^{\prime}$ of $B$ with $B=A^{\prime} \oplus R$.

Proof. These follow from Corollary 4.9 and the results of the third section.

\section{APpendix}

Two-sided modules. Thus far we have considered only one-sided $A$ modules although the two sided case would give more information concerning the structure of arbitrary Banach algebras. There are two reasons for making our restriction. First is the ability to determine for selected Banach algebras when all one- or two-dimensional cohomology groups vanish. Secondly in Wedderburn Theorem considerations when the superalgebra itself is commutative it suffices to consider just 1 -sided modules.

Now let $A$ be a Banach algebra and let $(M, \cdot)$ be a normed 2-sided $A$ module. That is, $(M, \cdot)$ is a 2-sided module in the usual sense and there exists $K>0$ such that for all $a \in A$ and $m \in M,\|a \cdot m\| \leqq K\|a\|\|m\|$ and $\|m \cdot a\| \leqq K\|a\|\|m\|$. Furthermore we assume that for all $a, b \in A$ and $m \in M$, $a \cdot(m \cdot b)=(a \cdot m) \cdot b$. Next define $\delta$, the coboundary map, by

$$
\begin{aligned}
\delta f\left(a_{1}, \cdots, a_{n+1}\right)= & a_{1} \cdot f\left(a_{2}, \cdots, a_{n+1}\right)+\sum_{i=1}^{n}(-1)^{i} f\left(a_{1}, \cdots, a_{i} a_{i+1}, \cdots, a_{n+1}\right) \\
& +(-1)^{n+1} f\left(a_{1}, \cdots, a_{n}\right) \cdot a_{n+1} .
\end{aligned}
$$

With this we can define bounded cocycles, coboundaries and cohomology groups in essentially the same manner as before with the assumption that a 1-cocycle (or derivation) $g$ is a coboundary provided there exists $m \in M$ such that $g(a)=a \cdot m-m \cdot a$ for all $a \in A$. The statement $H^{1}(A, M)=\{0\}$ means that every bounded derivation of $A$ in $M$ is inner.

If $A$ is an algebra and $(M, \cdot)$ is a 2 -sided $A$-module, then $(M, \cdot)$ is unital 
as a left (right) module if, and only if, $m \in M$ and $a \cdot m=0(m \cdot a=0)$ for all $a \in A$ imply $m=0$. Also $(M, \cdot)$ is a zero module when considered as a left (right) module if, and only if, $a \cdot m=0(m \cdot a=0)$ for all $a \in A$ and $m \in M$.

Let $A$ be a Banach algebra with 1 as identity and $(M, \cdot)$ a normed $A$ module. Consider the following four $A$-submodules of $(M, \cdot)$.

$$
\begin{aligned}
& M_{0,0}=\{m \in M \mid x \cdot m=m \cdot x \text { for all } x \in A\}, \\
& M_{0,1}=\{m \in M \mid x \cdot m=0 \text { for all } x \in A \text { and } m \cdot 1=m\}, \\
& M_{1,0}=\{m \in M \mid m \cdot x=0 \text { for all } x \in A \text { and } 1 \cdot m=m\}, \\
& M_{1,1}=\{m \in M \mid 1 \cdot m=m \cdot 1=m\} .
\end{aligned}
$$

Since $m=(m-1 \cdot m-m \cdot 1+1 \cdot m \cdot 1)+(1 \cdot m-1 \cdot m \cdot 1)+(m \cdot 1-1 \cdot m \cdot 1)$ $+1 \cdot m \cdot 1$, it is easy to show that $M=M_{0,0} \oplus M_{0,1} \oplus M_{1,0} \oplus M_{1,1}$. Also for all $k \in \omega, H^{k}(A, M)=H^{k}\left(A, M_{0,0}\right) \oplus H^{k}\left(A, M_{0,1}\right) \oplus H^{k}\left(A, M_{1,0}\right) \oplus H^{k}\left(A, M_{1,1}\right)$. Now if $k>1$ and $f$ is a bounded $\left(k, M_{0,1}\right)$ or $\left(k, M_{0,0}\right)$-cocycle of $A$, then letting $g\left(x_{1}, \cdots, x_{k-1}\right)=f\left(1, x_{1}, \cdots, x_{k-1}\right)$, we have $\delta g=f$. If $f$ is a bounded derivation of $A$ into $M_{0,1}$ or $M_{0,0}$, then if $m=f(1), f(x)=x \cdot m-m \cdot x$. Hence $H^{k}\left(A, M_{0,0}\right)=H^{k}\left(A, M_{0,1}\right)=\{0\}$. In a similar way $H^{k}\left(A, M_{1,0}\right)=\{0\}$ and hence $H^{k}(A, M)=H^{k}\left(A, M_{1,1}\right)$.

If $A$ has no identity, then adjoining the unit $e$ to $A$ and letting $(M, \cdot)$ be a normed $A_{e}$-module with $(x+\lambda e) \cdot m=x \cdot m+\lambda m$ and $m \cdot(x+\lambda e)=m \cdot x+\lambda m$ we find $(M, \cdot)$ is a unital normed $A_{e}$-module and as in Theorem 1.6, $H^{j}(A, M)$ $=\{0\}$ if, and only if, $H^{j}\left(A_{e}, M\right)=\{0\}, j=1,2$.

Let $A$ be a Banach algebra and $(M, \cdot)$ a normed $A$-module and suppose $S: A \times M \rightarrow M$ and $T: M \times A \rightarrow M$ are bounded bilinear functions which define the module, i.e., $S(a, m)=a \cdot m$ and $T(m, a)=m \cdot a$ for all $a \in A$ and $m \in M$. Then $S(a, T(m, b))=T(S(a, m), b)$ for all $a, b \in A$ and $m \in M$. Recall that we have defined in the previous section adjoint maps $S^{* * *}: A^{* *} \times M^{* *}$ $\rightarrow M^{* *}$ and $T^{* * *}: M^{* *} \times A^{* *} \rightarrow M^{* *}$. Using Theorem 3.2 of [1], the following statements can be verified:

(i) The adjoints $S^{* * *}$ and $T^{* * *}$ can be used to make $M^{* *}$ into a twosided normed $A^{* *}$-module $\left(M^{* *}, \cdot\right)$ which agrees with the usual module when restricted to $A$ and $M$.

(ii) The adjoints of one and two dimensional cocycles are again cocycles.

There is no problem in proving (i) and (ii) since in each term of the equations

$$
\begin{aligned}
a \cdot(m \cdot b) & =(a \cdot m) \cdot b, \\
a \cdot d(b)+d(a) \cdot b & =d(a b)
\end{aligned}
$$

and

$$
a \cdot f(b, c)-f(a b, c)+f(a, b c)-f(a, b) \cdot c=0,
$$

the variables appear in the same order. 
Now let $X$ be a compact Hausdorff space and $(M, \cdot)$ a normed $C(X)$ module which is unital as a left module and with $M$ finite dimensional. Then it is well known that there exist projections $P_{1}, \cdots, P_{n}$ on $M$ with $P_{i} P_{j}$ $=\delta_{i j} P_{i}$ and $\sum_{i=1}^{n} P_{i}=I$, the identity operator, and also points $s_{1}, \cdots, s_{n} \in X$ such that $u \cdot m=\sum_{i=1}^{n} u\left(s_{i}\right) P_{i}(m)$ for all $u \in C(X)$ and $m \in M$. If $(M, \cdot)$ is unital as a right module, then there exist $t_{1}, \cdots, t_{n} \in M$ with $m \cdot u$ $=\sum_{i=1}^{n} u\left(t_{i}\right) P_{i}(m)$.

For each $r \in X$, let $E(r)$ be the characteristic function of $\{r\}$. Then the map $\mu \rightarrow \int E(r) d \mu$ is a bounded linear functional on $C(X)^{*}$ and hence determines an element in $C(X)^{* *}$ which we shall call $e(r)$. Further for each $m \in M$, $e(r) \cdot m=0$ unless $r=s_{1}, \cdots, s_{n}$ and $e\left(s_{i}\right) \cdot m=P_{i}(m)$. Similar statements are valid on the right if $(M, \cdot)$ is unital as a right module.

Theorem A.1. Let $X$ be a compact Hausdorf space and $(M, \cdot)$ a normed $C(X)$-module. If $M$ is finite dimensional, then $H^{1}(C(X), M)=\{0\}$ and $H^{2}(C(X), M)=\{0\}$.

Proof. It suffices in either case to prove this when $(M, \cdot)$ is unital both as a right and left module. Let $s_{1}, \cdots, s_{n} \in X$ such that $u \cdot m=\sum_{i=1}^{n} u\left(s_{i}\right) P_{i}(m)$ $=\sum_{i=1}^{n} u\left(s_{i}\right) e\left(s_{i}\right) \cdot m$ for all $u \in C(X)$ and $m \in M$.

(i) Let $d$ be a bounded derivation of $C(X)$ into $M$. Let $e_{i}=e\left(s_{i}\right)$ and let $m=\sum_{i=1}^{n} e_{i} \cdot d^{* *}\left(e_{i}\right)$, where $d^{* *}$ is the double adjoint of $d$. Then

$$
x \cdot m-m \cdot x=x \cdot \sum_{i=1}^{n} e_{i} \cdot d^{* *}\left(e_{i}\right)-\sum_{i=1}^{n} e_{i} \cdot d^{* *}\left(e_{i}\right) \cdot x .
$$

Since $d^{* *}$ is a derivation of $C(X)^{* *}$ into $M$ and $x \cdot \sum_{i=1}^{n} e_{i} \cdot d^{* *}\left(e_{i}\right)=\sum_{i=1}^{n} x\left(s_{i}\right) e_{i}$ $\cdot d^{* *}\left(e_{i}\right)=\sum_{i=1}^{n} e_{i} \cdot d^{* *}\left(e_{i} x\right)=\sum_{i=1}^{n} e_{i} \cdot e_{i} \cdot d^{* *}(x)+\sum_{i=1}^{n} e_{i} \cdot d^{* *}\left(e_{i}\right) \cdot x$, we have

$$
x \cdot m-m \cdot x=\sum_{i=1}^{n} e_{i} \cdot d^{* *}(x)=d(x) .
$$

Thus every bounded derivation is inner.

(ii) Let $f$ be a bounded $(2, M)$-cocycle of $C(X)$ and $f^{* * *}$ its adjoint. Let $g(x)=\sum_{i=1}^{n} e_{i} \cdot f^{* * *}\left(e_{i}, x\right)$. Then

$$
\begin{aligned}
\delta g(x, y) & =x \cdot \sum_{i=1}^{n} e_{i} \cdot f^{* * *}\left(e_{i}, y\right)-\sum_{i=1}^{n} e_{i} \cdot f^{* * *}\left(e_{i}, x y\right)+\sum_{i=1}^{n} e_{i} \cdot f^{* * *}\left(e_{i}, x\right) \cdot y \\
& =\sum_{i=1}^{n} e_{i} \cdot f^{* * *}\left(e_{i} x, y\right)-\sum_{i=1}^{n} e_{i} \cdot f^{* * *}\left(e_{i}, x y\right)+\sum_{i=1}^{n} e_{i} \cdot f^{* * *}\left(e_{i}, x\right) \cdot y \\
& =\sum_{i=1}^{n} e_{i} \cdot f^{* * *}(x, y)=f^{* * *}(x, y)=f(x, y) .
\end{aligned}
$$

Thus $H^{2}(C(X), M)=\{0\}$. 
The connection between the Wedderburn principal theorem and cohomology groups is essentially the same as before. Thus we have the following:

THEOREM. Let $A$ be a commutative Banach algebra and $B$ a (not necessarily commutative) extension of $A$. Suppose $R$ is the radical of $B, R^{2}=\{0\}$ and $R$ is finite dimensional. If $H^{2}(A, M)=\{0\}$ for all finite dimensional $(M, \cdot)$, then there exists a closed subalgebra $A^{\prime}$ of $B$ with $B=A^{\prime} \oplus R$. If $H^{1}(A, M)=\{0\}$ for all finite dimensional $(M, \cdot)$ and if $B=A_{1} \oplus R=A_{2} \oplus R$, then $A_{1}$ and $A_{2}$ are quasi-inner automorphic, i.e. there exists an automorphism $\sigma$ of $A_{1}$ onto $A_{2}$ and $r \in R$ such that $\sigma(a)=a-a r+r a$ for all $a \in A_{1}$.

As before the theorem holds for arbitrary finite dimensional $R$.

TheOREM A.2. Let $B$ be a Banach algebra with finite dimensional radical $R$ satisfying $B / R=C(X)$ for some locally compact Hausdorff space $S$. Then there exists a closed subalgebra $C$ of $B$ with $B=C \oplus R$. If $B=C_{1} \oplus R=C_{2} \oplus R$, then $C_{1}$ and $C_{2}$ are quasi-inner-automorphic.

If $A$ is a semi-simple commutative Banach algebra without an identity, then there exists a module $(M, \cdot)$ such that $H^{1}(A, M) \neq\{0\}$. To this end let $M=A$ and define $x \cdot m=x m$ for $x \in A, m \in M$ and $m \cdot x=0$. Then the identity map $d$ on $A$ is a derivation of $A$ into $M$. But if $d(x)=x \cdot m-m \cdot x$ for some $m \in M$, then $x=x m$ for all $x \in A$, contradicting the statement that $A$ has no unit.

However it is an open question whether $H^{2}(C(X), M)=\{0\}$ for all normed $C(X)$-modules $(M, \cdot)$.

Added in proof. Theorem 4.1 can be improved to the following. If $X$ is a compact Hausdorff space and $(M, \cdot)$ is a one-sided $C(X)$-module, then there are no nontrivial derivations of $C(X)$ into $M$. To show this let $d$ be a derivation of $C(X)$ into $M$ and define a new norm $\||\|| \mid$ on the algebra of continuous functions on $X$ by $\|u\|\|=\| u\left\|_{\infty}+\right\| d(u) \|_{M}$. Then Theorem 4.1 of Bade and Curtis [Homomorphisms of commutative Banach algebras, Amer. J. Math. 82 (1960), 589-608] implies that $d$ is bounded (and hence equals 0 ) on some dense subalgebra $J(F)$ of the form $\left\{u \mid u \in C(X)\right.$ and $u(t) \equiv u\left(t_{i}\right)$ in a neighborhood of $\left.t_{i} \in F\right\}$ where $F$ is a finite subset of $X$. If $u=0$ in a neighborhood $V$ of $F$ and $v \in C(X)$, then $u$ and $u v \in J(F)$. Hence

$$
0=d(u v)=u \cdot d(v)+v \cdot d(u)=u \cdot d(v),
$$

and so $E_{X-F} \cdot d(v)=0$, where $E_{X-F}$ is the characteristic function of $X-F$. If $E_{F}$ is the characteristic function of $F$, then $E_{F} \cdot d(v)=0$, since $v \rightarrow E_{F} \cdot d(v)$ is essentially the sum of a finite number of point derivations. Thus $d(v)$ $=E_{\boldsymbol{F}} \cdot d(v)+E_{X-\boldsymbol{F}} \cdot d(v)=0$ for all $v \in C(X)$. 


\section{BIBLIOGRAPHY}

1. R. Arens, The adjoint of a bilinear operation, Proc. Amer. Math. Soc. 2 (1951), 839-848.

2. W. G. Bade and P. C. Curtis, The Wedderburn decomposition of commutative Banach algebras, Amer. J. Math. 82 (1960), 851-866.

3. N. Dunford and J. Schwartz, Linear operators. I, Interscience, New York, 1958.

4. C. F. Feldman, The Wedderburn principal theorem in Banach algebras, Proc. Amer. Math. Soc. 2 (1951), 771-777.

5. G. Hochschild, On the cohomology groups of an associative algebra, Ann. of Math. 46 (1945), 58-67

6. H. Kamowitz, Cohomology groups of commutative Banach algebras, Dissertation, Brown Univ., Providence, R.I., 1960 (unpublished).

7. L. H. Loomis, An introduction to abstract harmonic analysis, Van Nostrand, New York, 1953.

8. D. J. Newman, The non existence of projections from $L^{1}$ to $H^{1}$, Proc. Amer. Math. Soc. 12 (1961), 98-99.

9. A. Rosenberg and D. Zelinsky, Cohomology of infinite algebras, Trans. Amer. Math. Soc. 82 (1956), 85-98.

10. I. M. Singer and J. Wermer, Derivations on commutative normed algebras, Math. Ann. 129 (1955), 260-264.

11. D. Zelinsky, Raising idempotents, Duke Math J. 21 (1954), 315-322.

AVCO CORPORATION,

WiLMINGTON, MASSACHUSETTS 